\title{
Modeling Assumptions
}

The following tables detail the assumptions used in the model.

Table S1: Assumptions for processing times, arrival rates, batches and shifts

\begin{tabular}{|c|c|c|c|}
\hline Parameter & Assumption & Distribution & $\begin{array}{l}\text { Units for } \\
\text { Distribution }\end{array}$ \\
\hline School/workplace patient arrival rate & $\begin{array}{c}\text { Poisson process } \sim 9,240 \\
\text { arrivals/week }\end{array}$ & Poisson process $\sim 9,240$ & arrivals/week \\
\hline Community testing patient arrival rate & $\begin{array}{l}\text { Poisson process } \sim 9,240 \\
\text { arrivals/week }\end{array}$ & Poisson process $\sim 9,240$ & arrivals/week \\
\hline Nursing homes sample arrival rate & $\begin{array}{l}\text { Poisson process } \sim 8,400 \\
\text { arrivals/week }\end{array}$ & Poisson process $\sim 8,400$ & arrivals/week \\
\hline Match/Create EPIC record processing time & 4 minutes/patient & triangular $(3,4,5)$ & minutes/sample \\
\hline $\begin{array}{l}\text { Match Requisition (school/workplace) } \\
\text { processing time }\end{array}$ & $30 \mathrm{secs} /$ patient & triangular( $25,30,35)$ & seconds/sample \\
\hline $\begin{array}{c}\text { Sample Collection (school/workplace) } \\
\text { processing time }\end{array}$ & $30 \mathrm{secs} / \mathrm{sample}$ & triangular $(25,30,35)$ & seconds/sample \\
\hline $\begin{array}{c}\text { Sample Collection (community) processing } \\
\text { time }\end{array}$ & $30 \mathrm{secs} / \mathrm{sample}$ & triangular $(25,30,35)$ & seconds/sample \\
\hline $\begin{array}{l}\text { Sample Accessioning (rapid) (community) } \\
\text { processing time }\end{array}$ & $30 \mathrm{secs} / \mathrm{sample}$ & triangular( $25,30,35)$ & seconds/sample \\
\hline $\begin{array}{c}\text { Sample Accessioning (nursing homes) } \\
\text { processing time }\end{array}$ & 3-5 mins/sample & triangular $(3,5,6)$ & minutes/sample \\
\hline Manifest building processing time & 5 mins per 100 samples & triangular(3,3.5,5) & seconds/sample \\
\hline Rack Samples processing time & 1-2 secs /sample & triangular $(0,1,2)$ & seconds/sample \\
\hline Scan Barcode processing time & 30 secs/rack of 24 samples & triangular(24,30,36) & seconds/rack \\
\hline Uncapping processing time & 3 secs/sample & triangular(67.2,72,84) & seconds/rack \\
\hline Deswabbing processing time & 3-30 secs/sample & triangular(1.2,3,10) & minutes/rack \\
\hline STARlet processing time & 15 mins/plate of 96 samples & triangular( $14.5,15,15.5)$ & minutes/plate \\
\hline STAR processing time & $\begin{array}{l}1.5 \text { hours/ } 4 \text { x plates of } 96 \\
\text { samples }\end{array}$ & triangular(1.45,1.5,1.55) & hours/plate \\
\hline Mosquito processing time & $8 \mathrm{mins} / \mathrm{batch}$ & triangular( $7.8,8,8.5)$ & minutes/plate \\
\hline qPCR processing time & 1.25 hours/batch & triangular(1.24,1.25,1.3) & hours/batch \\
\hline
\end{tabular}




\begin{tabular}{|c|c|c|c|}
\hline Sample Retrieval processing time & 1.25 minutes / sample & triangular(1.1,1.25,1.3) & minutes/sample \\
\hline Export Data processing time & 4 mins/ batch & triangular( $3.5,4,4.5)$ & minutes/batch \\
\hline Manual Verification processing time & 15 secs/ sample & triangular( $(15,16,17)$ & seconds/sample \\
\hline Rack Sample batch size & 24 samples per batch & 24 & samples \\
\hline STARlet batch size & 96 samples per batch & 96 & samples \\
\hline STAR batch size & 384 samples per batch & 384 & samples \\
\hline qPCR batch size & 96 samples per batch & 96 & samples \\
\hline Shipping from Colleges batch size & 250 samples per batch & triangular(100,250,600) & samples \\
\hline Shipping form community testing batch size & 250 samples per batch & triangular $(100,250,600)$ & samples \\
\hline Positivity rate & $1.20 \%$ & 1.2 & percent \\
\hline Inconclusive test rate & $2.30 \%$ & 2.3 & percent \\
\hline Lab shift length & 8 hours & 8 & hours \\
\hline Accessioning shift length & 24 hours & 24 & hours \\
\hline
\end{tabular}


Table S2: Assumptions for number of resources

\begin{tabular}{|l|c|}
\hline Resource Capacities & Assumption \\
\hline Sample Accessioning (rapid) (community) & 33 technicians \\
Sample Accessioning (nursing homes) & 33 technicians \\
Manifest Building & 2 supervisors \\
Rack Samples & 2 technicians \\
Scan Barcode & 2 technicians \\
Uncapping & 2 technicians \\
Deswabbing & 2 technicians \\
STARlet & 3 instruments \\
STAR & 6 instruments \\
Mosquito & 3 instruments \\
qPCR & 16 instruments \\
\hline
\end{tabular}

